\title{
Spontaneous crystal coalescence enables highly efficient perovskite solar cells
}

Bart Roose, $\dagger^{a}$ Amita Ummadisingu, $\dagger^{b}$ Juan-Pablo Correa-Baena, ${ }^{c}$ Michael Saliba, ${ }^{b}$ Anders Hagfeldt, ${ }^{c}$ Michael Graetzel, ${ }^{b}$ Ullrich Steiner, ${ }^{a}$ Antonio Abate* a,d

aAdolphe Merkle Institute, Chemin des Verdiers 4, CH-1700 Fribourg, Switzerland

${ }^{\mathrm{b}}$ Laboratory of Photonics and Interfaces, Swiss Federal Institute of Technology (EPFL), Station 6, CH-1015 Lausanne, Switzerland

${ }^{\mathrm{c}}$ Laboratory of Photomolecular Science, Swiss Federal Institute of Technology (EPFL), Station 6, CH-1015 Lausanne, Switzerland

d Helmholtz-Zentrum Berlin für Materialien und Energie, Kekuléstrasse 5, 12489 Berlin, Germany

$\dagger$ Authors have contributed equally

*Corresponding author: antonio.abate@helmholtz-berlin.de antonioabate83@gmail.com

\begin{abstract}
Perovskite solar cells have recently reached staggering efficiencies, through efforts focused on reducing grain boundaries, by enlarging the size of the crystalline domains that constitute the perovskite films. Here, we demonstrate that smaller crystallites within perovskite films spontaneously coalesce into larger ones, even when complete devices are stored in the dark at room temperature. We show that crystal coalescence greatly improves the performance of state-of-the-art perovskite solar cells. Our results reveal the dynamic nature of the morphology of perovskite films and highlight the crucial role that coalescence plays in producing highly efficient devices.
\end{abstract}




\section{Graphical Abstract}

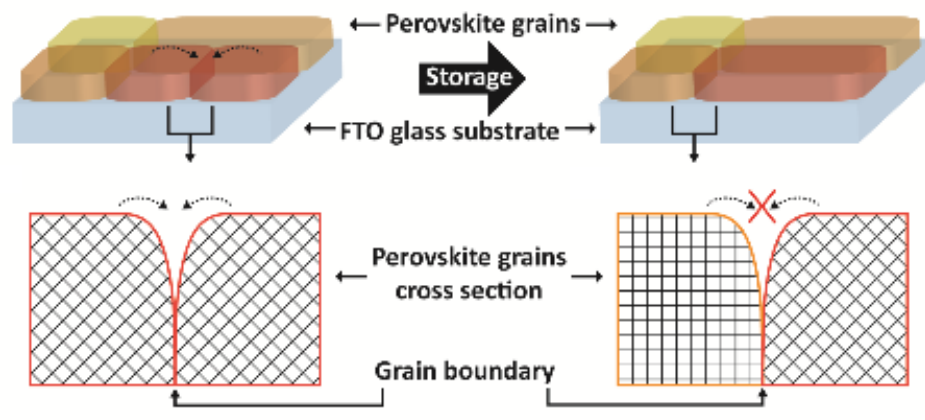

\section{Research Highlights}

- Perovskite crystals can coalesce during storage of full devices

- Larger crystals lead to increased performance and reduced hysteresis

- Coalescence may be an important reason for the extraordinary success of perovskite solar cells

Keywords: perovskite solar cell, photovoltaics, coalescence, aging, ion migration, hysteresis 


\section{Introduction}

Perovskite solar cells (PSCs) have rapidly become one of the most promising prototype technologies for low-cost and efficient harvesting of solar energy.[1-6] Recent reports demonstrated outstanding solar-to-electric power conversion efficiencies (PCEs) above 22\%.[7] Since the first demonstrations by Kim et al. [8] and Lee et al. [9], the main advances have been achieved through engineering the perovskite deposition, with the aim of forming a compact film with homogenous crystalline domains on the micrometre scale.[1,10] Several reports have indicated that small crystallites present between larger perovskite domains result in suboptimal charge extraction under stabilized working conditions, such as in maximum power point tracking, or in larger hysteresis under non-stabilized current-voltage $(J-V)$ scans.[11,12] Nevertheless, hysteresis is frequently found to decrease several hours to weeks after device preparation and is often accompanied by an increased stabilized PCE.[13-16] Improved performance or self-healing activated by light is well documented and it has been associated with the passivation of defects within the perovskite.[17,18] However, performance improvements have also been observed in devices stored in the dark at room temperature.[13] This is consistent with the common, and often undisclosed, practice of storing devices for several hours to a few days after preparation, and before measuring the first J-V curve.[19] The absence of any external influence suggests that a spontaneous mechanism is actively improving the devices during storage.

Here, we show that coalescence of small perovskite crystallites into larger crystalline domains takes place in perovskite films stored in dark at room temperature. Scanning electron microscopy (SEM) and X-ray diffraction (XRD) were employed to investigate the evolution of the perovskite film morphology. This study demonstrates that small perovskite crystallites in the film decrease in number and the average crystal size increases over the timescale of a few weeks. We have prepared state-of-the-art PSCs and then used time correlated single 
photon counting (TCSPC), intensity modulated photocurrent spectroscopy (IMPS) and photovoltaic performance measurements to demonstrate that perovskite crystal coalescence is a spontaneous, self-healing mechanism, which is inadvertently exploited to achieve high stabilized efficiencies.

\section{Material and methods}

\subsection{Scanning electron microscopy}

SEM micrographs were recorded on a Tescan MIRA 3 LMH with a field emission source operated at an acceleration voltage of $10 \mathrm{kV}$. SEM micrographs were further analysed using ImageJ.

\subsection{Time-correlated single-photon counting measurement}

Fluorescence lifetime data was collected using Florolog 322 spectrofluorometer (Horiba Jobin Ybon Ltd). A NanoLED-405LH (Horiba) laser diode (406 nm) (operated at a $400 \mathrm{kHz}$ repetition rate) was used for excitation. The samples were mounted at $60^{\circ}$ and the emission collected at $90^{\circ}$ from the incident beam path. The detection monochromator was set to 760 $\mathrm{nm}$ with a $14 \mathrm{~mm}$ slit width and the photoluminescence was recorded using a picosecond photodetection module (TBX-04, Horiba Scientific).

\section{$2.3 X$-ray diffraction}

X-ray diffraction was measured using a Rigaku Ultima IV with a $\mathrm{Cu} \mathrm{K} \alpha$ source $(\lambda=0.154060$

$\mathrm{nm}$ ) equipped with a dual position graphite diffracted beam monochromator and a scintillation counter detector, operating in the Bragg-Brentano geometry. A step size of $0.01^{\circ}$ was chosen and an acquisition time of $2 \mathrm{~min} /{ }^{\circ}$ was used for the measurements. A baseline correction was 
applied. The angle dependent instrumental broadening was determined using a $\mathrm{LaB}_{6}$ reference and subtracted from the full width at half maximum to determine the crystal size.

\subsection{Solar cell preparation}

Fluorine doped tin oxide coated glass slides (Sigma-Aldrich, $\sim 7 / \square$ ) were cleaned by sonication in 2\% Hellmanex solution for 15 minutes. After rinsing with deionised water and ethanol, the substrates were sonicated again with isopropanol and rinsed with acetone. The substrates were treated with UV-ozone for 15 minutes. For flat devices, a $\mathrm{SnO}_{2}$ compact layer was deposited by atomic layer deposition.[13] Mixed halide-mixed cation perovskite films were deposited from a precursor solution containing FAI (1 M), $\mathrm{PbI}_{2}(1.1 \mathrm{M}), \mathrm{MABr}(0.2 \mathrm{M})$, $\operatorname{PbBr}_{2}(0.2 \mathrm{M})$ and CsI (0.075 M) in anhydrous DMF:DMSO 4:1 (v:v). The perovskite solution was spin-coated using a two-step program, 1000 and $6000 \mathrm{rpm}$ for 10 and $20 \mathrm{~s}$ respectively. During the second step, $100 \mu \mathrm{l}$ of chlorobenzene was pipetted onto the spinning substrate 5 seconds before the end of the program. The substrates were then annealed at 100 ${ }^{\circ} \mathrm{C}$ for 1 hour in a nitrogen glove box. Subsequently, the substrates were cooled down for a few minutes and a Spiro-OMeTAD (Merck) solution (70 $\mathrm{mM}$ in chlorobenzene) doped with bis(trifluoromethylsulfonyl)imide lithium salt (Li-TFSI, Aldrich), tris(2-(1H-pyrazol-1-yl)-4tert-butylpyridine)-cobalt(III)tris(bis(tri uoromethylsulfonyl)imide) (FK209, Dyenamo) and 4tert-butylpyridine (TBP, Aldrich) was spun at $4000 \mathrm{rpm}$ for $20 \mathrm{~s}$. The molar ratio of additives for Spiro-OMeTAD was: $0.5,0.03$ and 3.3 for Li-TFSI, FK209 and TBP, respectively. Finally, $70 \mathrm{~nm}$ of gold was thermally evaporated under high vacuum on top of the HTM.

\subsection{Optoelectronic measurements}

For photovoltaic measurements, a solar simulator from ABET Technologies (Model 11016 Sun 2000) with a xenon arc lamp was used and the solar cell response was recorded using a 
Metrohm PGSTAT302N Autolab. The intensity of the solar simulator was calibrated to 100 $\mathrm{mW} / \mathrm{cm}^{2}$ using a silicon reference cell from ReRa Solutions (KG5 filtered). $J$ - $V$-curves were measured in reverse and forward bias at a scan rate of $10 \mathrm{mV} / \mathrm{s}$. A shadow mask was used do define the device active area (the area is given in the $J$ - $V$ figures). Maximum power point tracking was used to perform stability experiments. IMPS was performed according to the procedure described in literature, using a $625 \mathrm{~nm}$ or blue LED driver at short circuit conditions and a light intensity of $100 \mathrm{~mW} / \mathrm{cm}^{2}$ and a Metrohm PGSTAT302N Autolab.[12]

\section{Results and discussion}

\subsection{Photovoltaic performance}

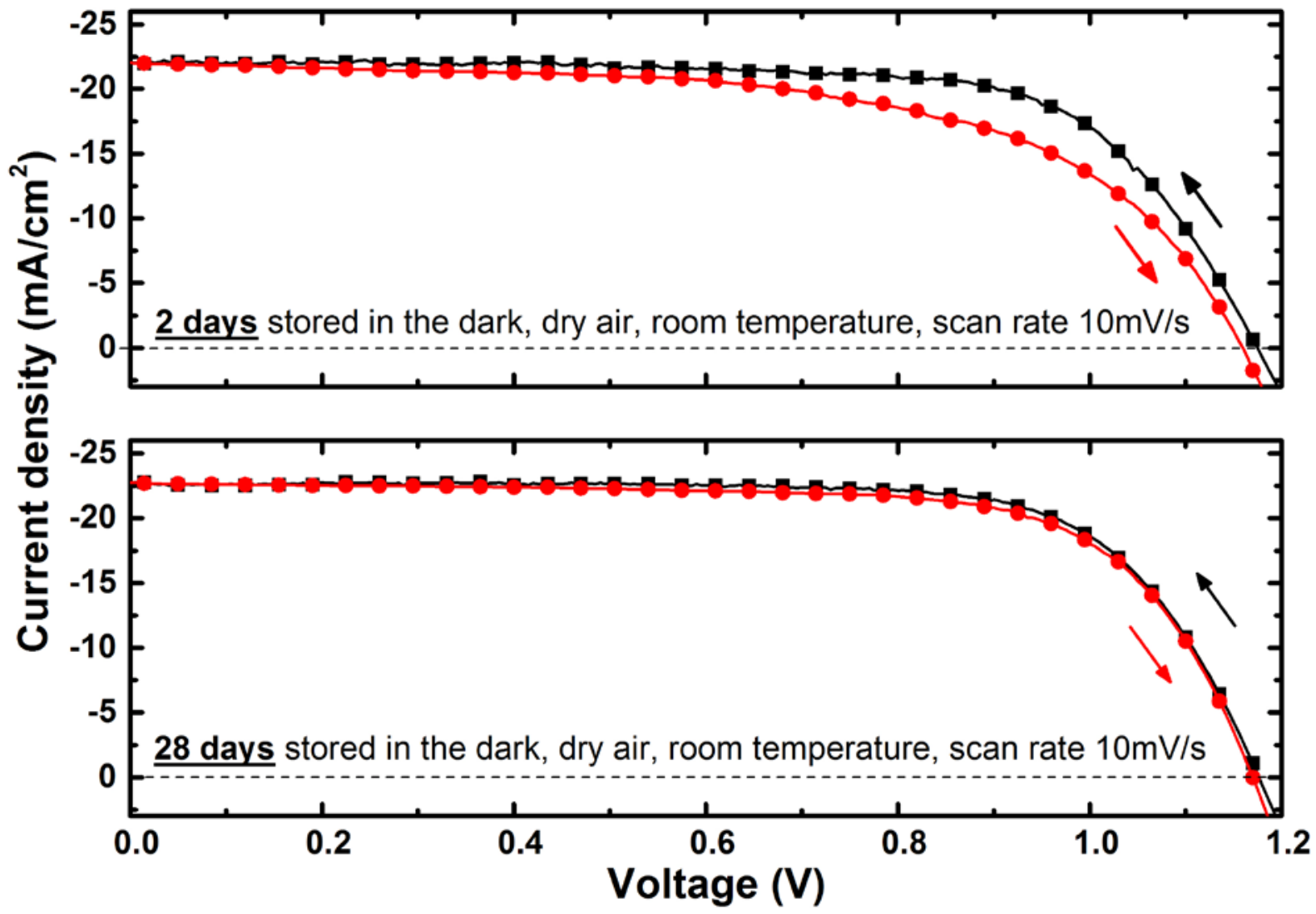

Figure 1. Current density-voltage $(J-V)$ curves for a perovskite solar cell at a 2 and $\mathbf{b} 28$ days after preparation, storing the device in the dark and in dry air at room temperature. The $J-V$ curves were measured at a scan rate of $10 \mathrm{mV} / \mathrm{s}$ from forward bias to short circuit condition and from short circuit condition to forward bias under AM1.5 simulated solar light illumination. The device was not preconditioned under light or voltage bias before each $J-V$ scan. The active area was defined by a shadow mask with an aperture of $0.16 \mathrm{~cm}^{2}$. 


\begin{tabular}{|c|c|c|c|c|c|c|}
\hline & $\begin{array}{c}\text { Scan } \\
\text { direction }\end{array}$ & $\begin{array}{l}V_{\text {oc }} \\
(\mathrm{V})\end{array}$ & $\begin{array}{c}J_{\mathrm{sc}} \\
\left(\mathrm{mA} / \mathbf{c m}^{2}\right)\end{array}$ & $\begin{array}{l}\text { FF } \\
(\%)\end{array}$ & $\begin{array}{l}\text { PCE } \\
(\%)\end{array}$ & $\begin{array}{l}\text { Light intensity } \\
\left(\mathbf{m W} / \mathbf{c m}^{2}\right)\end{array}$ \\
\hline \multirow{2}{*}{2 days } & FB to SC & 1.175 & 22.0 & 70.3 & 18.7 & \multirow{2}{*}{97.1} \\
\hline & $\mathrm{SC}$ to $\mathrm{FB}$ & 1.159 & 21.9 & 59.5 & 15.6 & \\
\hline \multirow{2}{*}{28 days } & $\mathrm{FB}$ to $\mathrm{SC}$ & 1.176 & 22.7 & 72.5 & 19.3 & \multirow{2}{*}{100.5} \\
\hline & $\mathrm{SC}$ to $\mathrm{FB}$ & 1.170 & 22.7 & 70.6 & 18.7 & \\
\hline
\end{tabular}

Table 1. Photovoltaic performance parameters: open-circuit voltage $\left(V_{\text {oc }}\right)$, short circuit current $\left(J_{\mathrm{sc}}\right)$, fill factor $(\mathrm{FF})$ and maximum power conversion efficiency (PCE) extracted from the $J$ - $V$ curves in Figure 1, from forward bias (FB) to short circuit (SC) and back.

We prepared state-of-the-art PSCs in an inert atmosphere, using a lead-based mixed halide (bromine and iodine) and cation (methylammonium and formamidinium) perovskite in a planar device architecture, as reported in the most recent literature.[20] Figure 1 displays the current density-voltage (J-V) curves of the same PSC collected under identical conditions, 2 days and again 28 days after preparation and storage in dry air (below 1\% relative humidity) at room temperature in the dark. The device performance parameters are listed in Table 1. It is evident that hysteresis is reduced and performance has improved with storage. As previously reported, we observed the opposite trend, i.e. reduced performances and improved hysteresis, when $J$-Vs were collected every day or when devices were stored in ambient light.[7] The PCE can increase by up to $25 \%$ after a few days or weeks of storage in the dark (see SI). Interestingly, devices with an initial lower PCE show more improvement and they tend to come closer to the highest performing ones. Having eliminated the influences of light, water and applied voltage during storage, oxygen remains as the only external agent that may potentially affect the device performance. Oxygen is known to interact with metal oxides, organic semiconductors and the perovskite employed in these devices and modifies their electronic properties.[21-25] However, oxygen permeation within the device takes place on the timescale of a few hours and is likely to saturate before the first $J-V$ curve is collected.[23,26,27] Therefore, oxygen and other external stimuli can be ruled out as playing an important role in the improvement of PCEs during storage in the dark at room temperature. 


\subsection{Optoelectronic characterization}
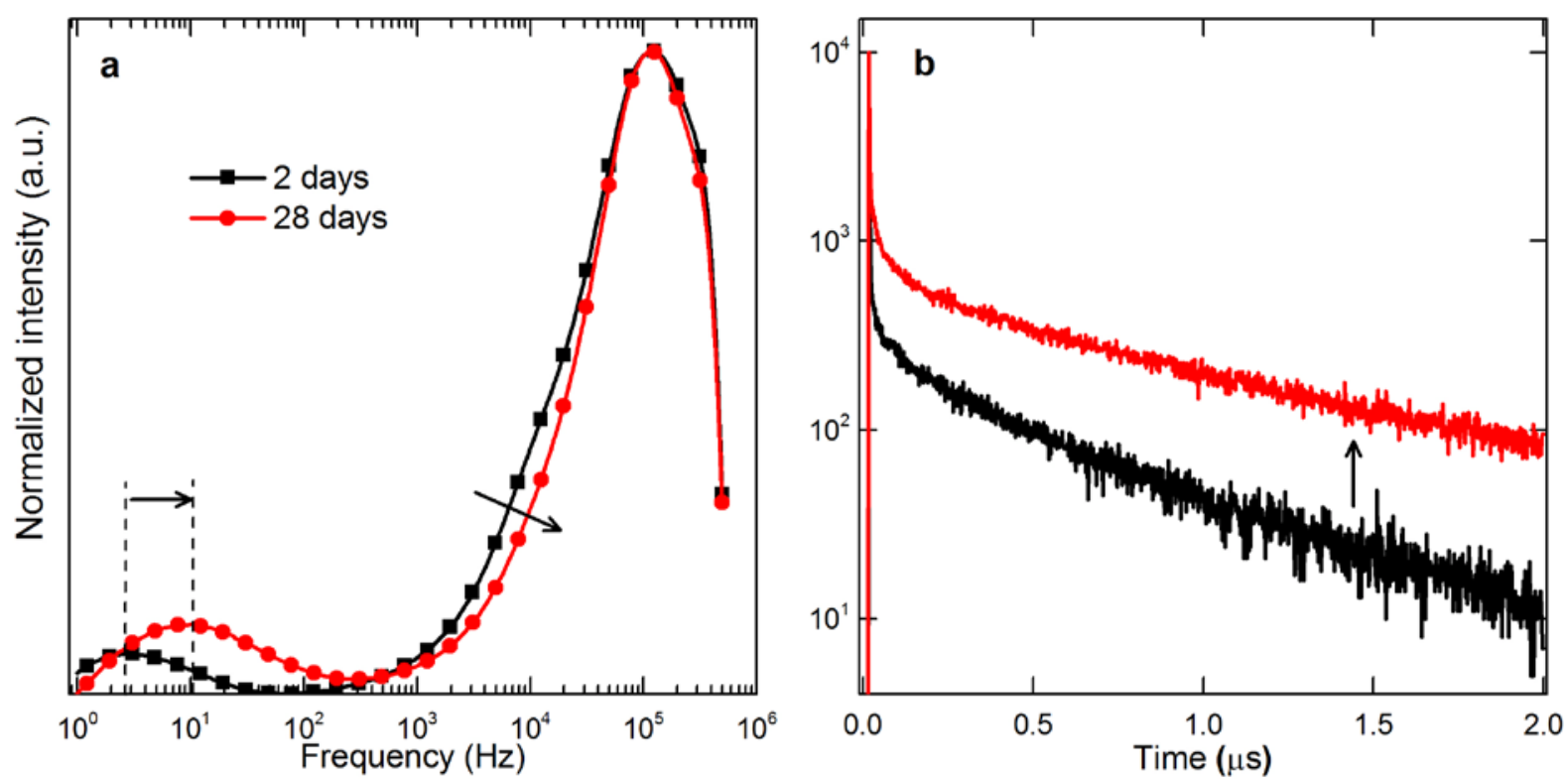

Figure 2. Data collected 2 and 28 days after the sample preparation. The samples were stored in the dark and in dry air at room temperature. a Imaginary part of the intensity modulated photocurrent spectra of a complete PSC. The spectra were normalized to the highest peak at $10^{5} \mathrm{~Hz}$. b Time-correlated single-photon counting measurement of a perovskite film deposited on a microscope glass slide. The sample was excited at $480 \mathrm{~nm}$ from the perovskite side and the emission at $760 \mathrm{~nm}$ from the same side was monitored.

To shed light on the origin of the PCE improvement due to dark storage, we performed intensity modulated photocurrent spectroscopy (IMPS) on a complete device. Figure 2a shows the imaginary component frequency spectra of the current response to the light modulated intensity (10\% of the stationary value) around $100 \mathrm{~mW} \mathrm{~cm}^{-2}$, collected from the same device 2 and 28 days after preparation. The spectra show three main features, which have been reported earlier by Correa-Baena et al. and Seo et al. for similar PSCs.[11,12] The feature at low frequencies $\left(10^{0}-10^{2} \mathrm{~Hz}\right)$ stems from the resonant frequencies of ion and ion vacancy migration within the perovskite lattice.[11,12,28] Here, we note that this feature is shifted towards higher frequencies or faster responses in the spectrum for the 28 day old sample. According to the study of Correa-Baena et al.,[11] a faster ionic response is indicative of larger crystalline domains within the perovskite film. Moving to higher frequencies, there are two more features that have been assigned to the resonant frequencies 
of the charges within the perovskite film and the other device components, such as the hole and the electron transporting layers. While the peak at the highest frequency $\left(10^{5} \mathrm{~Hz}\right)$ is unaffected, the shoulder at $10^{4} \mathrm{~Hz}$ is less pronounced after a long period of storage in the dark. Seo et al. demonstrated that this behaviour is correlated to a reduced concentration of smaller crystallites within the perovskite film, which results in better device performance.[12] To further investigate the changes in the perovskite film due to storage in the dark, we performed time-correlated single-photon counting measurements. Figure $2 \mathrm{~b}$ displays decay traces measured 2 and 28 days after preparation of the perovskite film, which was deposited on a glass microscope slide. An exponential function was fitted to the data between 0.15 and $1.95 \mu$ s and the photoluminescence decay time was indeed found to be significantly longer after 28 days of storage in the dark and dry air $(0.64 \mu \mathrm{s})$ compared to that after 2 days $(0.45 \mu \mathrm{s})$ (see SI for details). This points to a reduction in the non-radiative recombination through electronic defects or trap states within the perovskite film.[29] We have excluded from our experiment any external agent capable of passivating defects, such as water and light,[30] therefore the presence of increased crystalline domain sizes, suggested by IMPS, appears to be the most plausible explanation for the increased photoluminescence lifetime. As defects are more numerous at or near grain boundaries,[31,32] the number of trap states is greatly reduced by enlarging the crystalline domains within the perovskite film.

Therefore, the optoelectronic characterization suggests that storing the PSCs under dry conditions in the dark for a few weeks results in reduced non-radiative charge recombination and improved charge transport, which may be correlated to morphological changes in the perovskite film. 
a)

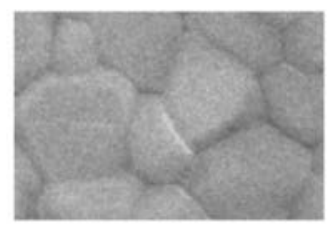

$100 \mathrm{~nm}$ b)

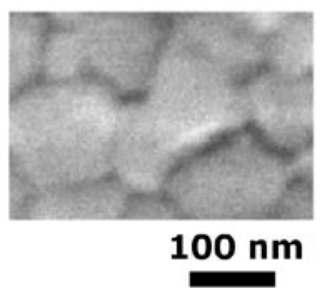

c)

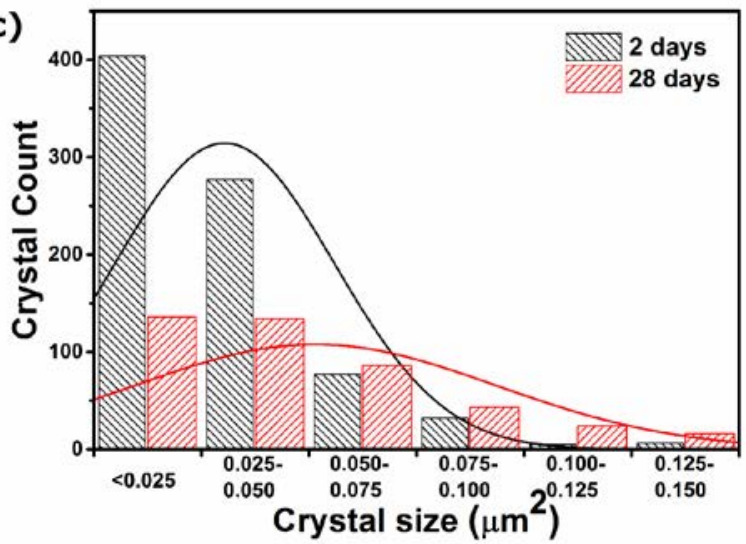

d)
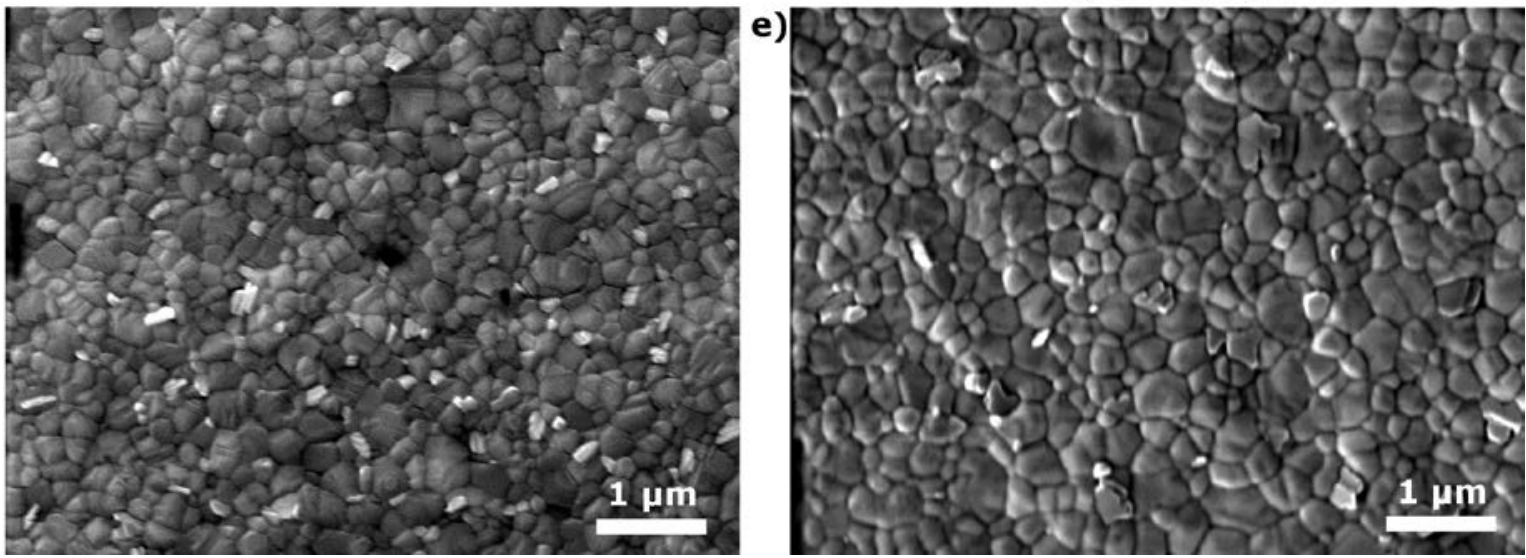

Figure 3. Scanning electron microscopy analysis of a perovskite film stored for a 2 and $\mathbf{b} 28$ days in dark and dry air at room temperature. c Statistical distribution of the area of the crystalline domains from the SEM top view in $\mathbf{d}$ (2 days) and $\mathbf{e}$ (28 days).

We studied the morphological evolution of the perovskite film by top view scanning electron microscopy (SEM) analysis. The images were collected from the same area of the film 2 days (Figure 3a) and 28 days after preparation and storage (Figure 3b) at room temperature in the dark under dry conditions. Two adjacent crystals in the centre of Figure 3a have merged into a larger single crystalline domain in Figure 3b, eliminating one grain boundary. To show the statistical relevance of this effect and to exclude electron beam induced changes to the surface, the distribution of grain areas (Figure 3c) was extracted from larger SEM top view images (Figure 3d and e) that were collected several $\mu \mathrm{m}$ apart. A statistical analysis revealed a significant reduction in the number of small crystallites (i.e. $<0.025 \mu \mathrm{m}^{2}$ ). We found a $66 \%$ lower number of grains per surface area and the average crystal size almost doubles after 28 
days storage. More detailed data is presented in the SI, including the size distribution for a sample aged for 14 days. We attribute this observation to the phenomenon of coalescence, where adjoining crystals fuse to form larger crystals. This phenomenon has been reported to take place in thin films of various materials including metals,[33] and polymers[34] during processes such as aging and sintering.[35] Solvent annealing[36] and Ostwald ripening[37] were systematically exploited to increase the average crystal size and thus the photovoltaic performance in PSCs.[1] Recently, Sheng et al. also reported spontaneous increase of the average crystal size in $\mathrm{CH}_{3} \mathrm{NH}_{3} \mathrm{PbBr}_{3}$ perovskite film stored in the dark at room temperature, but a potential impact on PSCs performance remained unexplored.[38]

In order to verify that coalescence can occur in a full device, where a layer of Spiro-OMeTAD covers the perovskite film, additional SEM images were collected before depositing the SpiroOMeTAD and washing off the Spiro-OMeTAD after ageing (see SI). It was found that coalescence indeed occurs in a complete device.

To prove the observed increase in crystal size in SEM micrographs is not a surface effect, XRD measurements were performed, which provide information about the bulk of the film. XRD measurements of the perovskite film 2 and 28 days after preparation are shown in Figure 4. All peaks can be assigned to the tetragonal perovskite structure, $\mathrm{PbI}_{2}$ excess in the perovskite film and FTO from the substrate.[39] The Miller indices, the relative intensity of the peaks and the extracted crystal sizes are given in Table 2. The average domain size of perovskite crystals was extracted two and 28 days after preparation, by subtracting the instrumental broadening and using the Scherrer equation.[40] For most of the crystal orientations shown in Table 2, the domain size significantly increased after storage in the dark. In accordance with the SEM results, it is found that the average crystal size is almost 
doubled on average. The changes in relative peak intensities suggests the preferential growth of certain crystal orientations over others, which may affect the device performances.

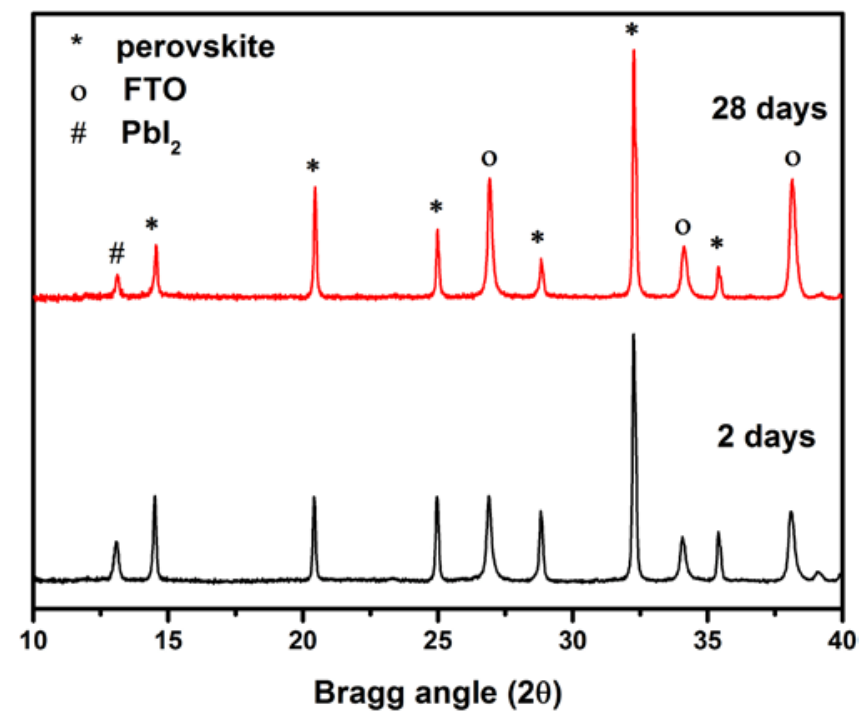

Figure 4: X-ray diffraction patterns collected 2 and 28 days after perovskite film preparation. The samples were stored in the dark and in dry air at room temperature.

\begin{tabular}{cccccc}
$\begin{array}{c}\text { Angle } \\
(\mathbf{2 \theta})\end{array}$ & hkl & $\begin{array}{c}\text { Intensity } \\
\text { 2 days }\end{array}$ & $\begin{array}{c}\text { Intensity } \\
\mathbf{2 8} \text { days }\end{array}$ & $\begin{array}{c}\text { Size } \\
\mathbf{2} \text { days } \\
\text { (nm) }\end{array}$ & $\begin{array}{c}\text { Size } \\
\mathbf{2 8} \text { days } \\
\text { (nm) }\end{array}$ \\
\hline 14.5 & 002,110 & 0.34 & 0.21 & $270 \pm 10$ & $380 \pm 10$ \\
20.4 & 112,020 & 0.34 & 0.44 & $440 \pm 10$ & $840 \pm 40$ \\
25.0 & 022 & 0.34 & 0.27 & $340 \pm 10$ & $1400 \pm 100$ \\
28.8 & 004,220 & 0.28 & 0.16 & $330 \pm 10$ & $340 \pm 10$ \\
32.3 & $114,222,130$ & 1.00 & 1.00 & $300 \pm 10$ & $380 \pm 10$ \\
35.4 & 024,132 & 0.20 & 0.12 & $160 \pm 10$ & $190 \pm 10$ \\
\hline
\end{tabular}

Table 2. Miller indices $(\mathrm{hkl})$, peak intensity normalized to the intensity of the perovskite peak at $32.32 \theta$ and the crystal size extracted from the spectra in Figure 4.

\subsection{Thermodynamics of coalescence}

The grain-grain orientation is of importance for coalescence, since the surface energy associated with each grain boundary varies based on the crystallographic misorientation between the contacting faces.[34] If the degree of misorientation at the grain boundary is low, the boundary energy is also low and these crystals preferentially coalesce, as shown in the schematic in Figure 5a and 5c. On the other hand, a high degree of misorientation at the grain boundary, as shown in Figure $5 b$ and $5 d$, is unfavourable for coalescence. This explains the directional selectivity of coalescence shown in Table 2. On this basis, we distinguish 
coalescence from the Ostwald ripening phenomena reported in literature,[38] which also causes the average size of perovskite crystals to increase. Ostwald ripening involves the transfer of material from the smaller crystals to the larger ones through an external phase which acts as a transfer medium (for instance, 2-propanol solution [38]). In contrast, coalescence involves the selective fusion of adjacent crystals by removing a connecting grain boundary.

a

b

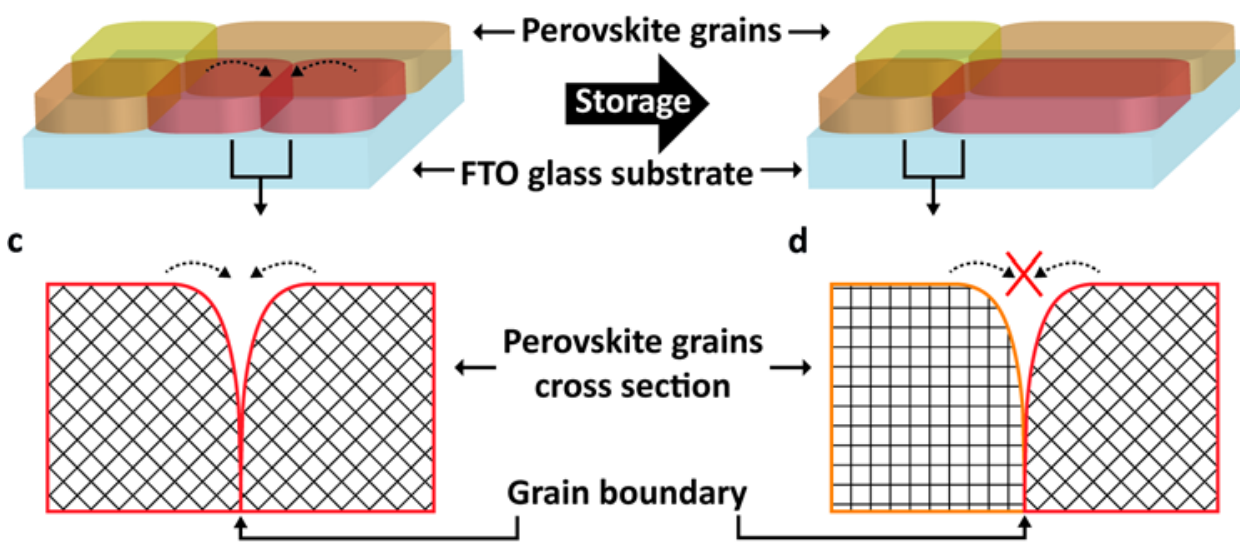

Figure 5. Schematic of crystal coalescence in a perovskite film. a An as-prepared film of crystals with a small crystallographic misorientation at the grain boundary shown in red. Arrows indicate the grain boundary of interest between these crystals. b Film after dark storage, showing the coalescence of these crystals. c Cross sectional view of crystals with a small crystallographic misorientation at the grain boundary which preferentially coalesce. d Cross sectional view of crystals with a large crystallographic misorientation at the grain boundary which do not coalesce.

\section{Conclusions}

We report on the coalescence of perovskite crystals in PSCs stored in dark at room temperature, showing that perovskite films within complete devices are highly dynamic, where small crystals spontaneously coalesce to form larger crystals. This brings about a reduction in the number of grain boundaries and the associated trap states, which suppresses non-radiative recombination, resulting in increased PCE and reduced hysteresis upon storage. This self-repair ability, which improves the electronic properties of perovskite films, provides an important insight into the success of perovskites as photovoltaic materials. 


\section{Author Contributions}

B.R., A.U and A.A. designed the study. B.R. and A.U. wrote the first version of the paper with inputs from A.A. B.R prepared perovskite films, measured the XRD spectra, collected and analysed the SEM images and the IMPS spectra. B.R. and A.U. analysed the XRD data. A.U. developed the thermodynamic aspects, measured and analysed the TCSPC data. B.R., J.C and M.S. prepared and characterized solar cells. A.H supervised J.C., M.G. supervised A.U. and U.S. supervised B.R. All authors commented on the manuscript.

\section{Acknowledgements}

This work was supported by the Swiss National Science Foundation [grant number 200021157135/1]; the King Abdulaziz City for Science and Technology; the Adolphe Merkle Foundation; and the Swiss National Science Foundation [Program NRP70 No. 153990]. 


\section{References}

[1] W. Nie, H. Tsai, R. Asadpour, J.-C. Blancon, A. J. Neukirch, G. Gupta, J. J. Crochet, M. Chhowalla, S. Tretiak, M. A. Alam, Science 347 (2015) 522-525.

[2] M. Sessolo, H.J. Bolink, Science 350 (2015) 917.

[3] M. Saliba, T. Matsui, K. Domanski, J.-Y. Seo, A. Ummadisingu, S.M. Zakeeruddin, J.-P. CorreaBaena, W.R. Tress, A. Abate, A. Hagfeldt, Science 354 (2016) 206-209.

[4] L. M. Pazos-Outón, M. Szumilo, R. Lamboll, J.M. Richter, M. Crespo-Quesada, M. Abdi-Jalebi, H.J. Beeson, M. Vrućinić, M. Alsari, H.J. Snaith, Science 351 (2016) 1430-1433.

[5] D.P. McMeekin, G. Sadoughi, W. Rehman, G.E. Eperon, M. Saliba, M.T. Hörantner, A. Haghighirad, N. Sakai, L. Korte, B. Rech, Science 351 (2016) 151-155.

[6] G.E. Eperon, T. Leijtens, K. A. Bush, R. Prasanna, T. Green, J. T.-W. Wang, D. P. McMeekin, G. Volonakis, R. L. Milot, R. May, A. Palmstrom, D.J. Slotcavage, R.A. Belisle, J.B. Patel, E.S. Parrott, R.J. Sutton, W. Ma, F. Moghadan, B. Conings, A. Babayigit, H.G. Boyen, S. Bent, F. Giustino, L.M. Herz, M.B. Johnston, M.D. McGehee, H.J. Snaith, Science 354 (2016) 861-865.

[7] N.-G. Park, M. Grätzel, T. Miyasaka, K. Zhu, K. Emery, Nat. Energy 1 (2016) 16152.

[8] H.-S. Kim, C.-R. Lee, J.-H. Im, K.-B. Lee, T. Moehl, A. Marchioro, S.-J. Moon, R. Humphry-Baker, J.H. Yum, J.E. Moser, Sci. Rep. 2 (2012) 591.

[9] M.M. Lee, J. Teuscher, T. Miyasaka, T.N. Murakami, H.J. Snaith, Science 338 (2012) 643-647. [10] N.K. Noel, S.N. Habisreutinger, B. Wenger, M.T. Klug, M.T. Hörantner, M.B. Johnston, R.J. Nicholas, D.T. Moore, H.J. Snaith, Energy Environ. Sci. 10 (2017) 145-152.

[11] J.P. Correa-Baena, M. Anaya, G. Lozano, W. Tress, K. Domanski, M. Saliba, T. Matsui, T.J. Jacobsson, M.E. Calvo, A. Abate, M. Grätzel, H. Miguez, A. Hagfeldt, Adv. Mater. 28 (2016) 50315037.

[12] J.Y. Seo, T. Matsui, J. Luo, J.P. Correa-Baena, F. Giordano, M. Saliba, K. Schenk, A. Ummadisingu, K. Domanski, M. Hadadian, A. Hagfeldt, S.M. Zakeeruddin, U. Steiner, M. Grätzel, A. Abate, Adv. Energy Mater. 6 (2016) 1600767.

[13] J.P.C. Baena, L. Steier, W. Tress, M. Saliba, S. Neutzner, T. Matsui, F. Giordano, T.J. Jacobsson, A.R.S. Kandada, S.M. Zakeeruddin, A. Petrozza, A. Abate, M.K. Nazeeruddin, M. Grätzel, A. Hagfeldt, Energy Environ. Sci. 8 (2015) 2928-2934.

[14] M.-C. Kim, B.J. Kim, D.-Y. Son, N.-G. Park, H.S. Jung, M. Choi, Nano Lett. 16 (2016) 5756-5763.

[15] J.H. Noh, S.H. Im, J.H. Heo, T.N. Mandal, S.I. Seok, Nano Lett. 13 (2013) 1764-1769.

[16] J. Song, E. Zheng, J. Bian, X.-F. Wang, W. Tian, Y. Sanehira, T. Miyasaka, J. Mater. Chem. A 3 (2015) 10837-10847.

[17] W. Zhang, V.M. Burlakov, D.J. Graham, T. Leijtens, A. Osherov, V. Bulović, H.J. Snaith, D.S. Ginger, S.D. Stranks, Nat. Commun. 7 (2016), 11683.

[18] C. Zhao, B. Chen, X. Qiao, L. Luan, K. Lu, B. Hu, Adv. Energy Mater. 5 (2015) 1500279.

[19] A. Abate, S. Paek, F. Giordano, J.-P. Correa-Baena, M. Saliba, P. Gao, T. Matsui, J. Ko, S.M.

Zakeeruddin, K. H. Dahmen, A. Hagfeldt, M. Grätzel, M.K. Nazeeruddin, Energy Environ. Sci. 8 (2015) 2946-2953.

[20] E.H. Anaraki, A. Kermanpur, L. Steier, K. Domanski, T. Matsui, W. Tress, M. Saliba, A. Abate, M. Grätzel, A. Hagfeldt, Energy Environ. Sci. 9 (2016) 3128-3134.

[21] A. Abate, T. Leijtens, S. Pathak, J. Teuscher, R. Avolio, M.E. Errico, J. Kirkpatrik, J.M. Ball, P. Docampo, I. McPherson, H.J. Snaith, Phys. Chem. Chem. Phys. 15 (2013) 2572-2579.

[22] T. Leijtens, G E. Eperon, S. Pathak, A. Abate, M.M. Lee, H.J. Snaith, Nat. Commun. 4 (2013) 2885. [23] N. Aristidou, I. Sanchez-Molina, T. Chotchuangchutchaval, M. Brown, L. Martinez, T. Rath, S.A. Haque, Angew.Chem.Int. Ed. 54 (2015) 8208-8212.

[24] F. T. O'Mahony, Y.H. Lee, C. Jellett, S. Dmitrov, D.T. Bryant, J.R. Durrant, B.C. O'Regan, M. Grätzel, M.K. Nazeeruddin, S.A. Haque, J. Mater. Chem. A 3 (2015) 7219-7223.

[25] A.D. Sheikh, A. Bera, M.A. Haque, R.B. Rakhi, S. Del Gobbo, H.N. Alshareef, T. Wu, Sol. Energ. Mater. Sol. Cells 137 (2015) 6-14. 
[26] H.-H. Fang, S. Adjokatse, H. Wei, J. Yang, G.R. Blake, J. Huang, J. Even, M.A. Loi, Sci. Adv. 2 (2016) 1600534.

[27] L. Contreras, J. Idígoras, A. Todinova, M. Salado, S. Kazim, S. Ahmad, J.A. Anta, Phys.Chem. Chem. Phys. 18 (2016) 31033-31042.

[28] X. Li, D. Bi, C. Yi, J.-D. Décoppet, J. Luo, S.M. Zakeeruddin, A. Hagfeldt, M. Grätzel, Science 353 (2016) 58-62.

[29] N.K. Noel, A. Abate, S.D. Stranks, E.S. Parrott, V.M. Burlakov, A. Goriely, H.J. Snaith, ACS Nano 8 (2014) 9815-9821.

[30] D.W. de Quilettes, S.M. Vorpahl, S.D. Stranks, H. Nagaoka, G.E. Eperon, M.E. Ziffer, H.J. Snaith, D.S. Ginger, Science 348 (2015) 683-686

[31] M. Pattabi, N. Suresh, S.M. Chaudhari, A. Banerjee, D.M. Phase, A. Gupta, K.M. Rao, Thin Solid Films 322 (1998) 340-343.

[32] G.G. Paulson, A.L. Friedberg, Thin Solid Films 5 (1970) 47-62.

[33] A. Goudy, M.L. Gee, S. Biggs, S. Underwood, Langmuir 11 (1995) 4454-4459.

[34] R. German, Sintering: from empirical observations to scientific principles, ButterworthHeinemann, Oxford, 2014.

[35] D. Liu, L. Wu, C. Li, S. Ren, J. Zhang, W. Li, L. Feng, ACS Appl. Mater. Interfaces 7 (2015) 1633016337.

[36] J. Liu, C. Gao, X. He, Q. Ye, L. Ouyang, D. Zhuang, C. Liao, J. Mei, W. Lau, ACS Appl. Mater. Interfaces 7 (2015) 24008-24015.

[37] M. Yang, T. Zhang, P. Schulz, Z. Li, G. Li, D.H. Kim, N. Guo, J.J. Berry, K. Zhu, Y. Zhao, Nat. Commun. 7 (2016) 12305.

[38] R. Sheng, X. Wen, S. Huang, X. Hao, S. Chen, Y. Jiang, X. Deng, M.A. Green, A.W.Y. Ho-Baillie, Nanoscale 8 (2016) 1926-1931.

[39] T.J. Jacobsson, L.J. Schwan, M. Ottosson, A. Hagfeldt, T. Edvinsson, Inorg. Chem. 54 (2015) 10678-10685.

[40] A.L. Patterson, Phys. Rev. 56 (1939) 978. 